\title{
Is an integrative laboratory algorithm more effective in detecting alpha-1-antitrypsin deficiency in patients with premature chronic obstructive pulmonary disease than AAT concentration based screening approach?
}

Andjelo Beletic*1 , Aleksandra Dudvarski-llic ${ }^{2}$, Branislava Milenkovic ${ }^{2}$, Ljudmila Nagorni-Obradovic ${ }^{2}$, Mila Ljujic ${ }^{3}$, Valentina Djordjevic ${ }^{3}$, Dusko Mirkovic ${ }^{4}$, Dragica Radojkovic ${ }^{3}$, Nada Majkic-Singh ${ }^{5}$

${ }^{1}$ Center for Medical Biochemistry, Clinical Center of Serbia, Belgrade, Serbia

${ }^{2}$ School of Medicine, University of Belgrade \& Clinic for Lung Diseases, Clinical Center of Serbia, Belgrade, Serbia

${ }^{3}$ Institute of Molecular Genetics and Genetic Engineering, University of Belgrade, Serbia

${ }^{4}$ Faculty of Pharmacy, University of Belgrade \& Center for Medical Biochemistry, Clinical Center of Serbia, Belgrade, Serbia

${ }^{5}$ Society of Medical Biochemists of Serbia, Belgrade, Serbia

*Corresponding author: andjelo.beletic@yahoo.com

\begin{abstract}
Introduction: Alpha-1-antitrypsin deficiency (AATD), genetic risk factor for premature chronic obstructive pulmonary disease (COPD), often remains undetected. The aim of our study was to analyse the effectiveness of an integrative laboratory algorithm for AATD detection in patients diagnosed with COPD by the age of 45 years, in comparison with the screening approach based on AAT concentration measurement alone.

Subjects and methods: 50 unrelated patients ( 28 males / 22 females, age 52 (24-75 years) diagnosed with COPD before the age of 45 years were enrolled. Immunonephelometric assay for alpha-1-antitrypsin (AAT) and PCR-reverse hybridization for $Z$ and $S$ allele were first-line, and isoelectric focusing and DNA sequencing (ABI Prism BigDye) were reflex tests.

Results: AATD associated genotypes were detected in 7 patients $\left(5 \mathrm{ZZ}, 1 \mathrm{ZM}_{\text {malton, }}, \mathrm{ZOO}_{\text {amersfoort }}\right), 10$ were heterozygous carriers $(8 \mathrm{MZ}$ and $2 \mathrm{MS}$ genotypes) and 33 were without AATD (MM genotype). Carriers and patients without AATD had comparable AAT concentrations ( $P=0.125)$. In majority of participants (48) first line tests were sufficient to analyze AATD presence. In two remaining cases reflex tests identified rare alleles, $M_{\text {malton }}$ and $\mathrm{QO}_{\text {amersfoort }}$ the later one being reported for the first time in Serbian population. Detection rate did not differ between algorithm and screening both for AATD $(P=0.500)$ and carriers $(P=0.063)$.

Conclusion: There is a high prevalence of AATD affected subjects and carriers in a group of patients with premature COPD. The use of integrative laboratory algorithm does not improve the effectiveness of AATD detection in comparison with the screening based on AAT concentration alone.
\end{abstract}

Key words: alpha-1-antitrypsin deficiency; diagnostic techniques and procedures; pulmonary disease, chronic obstructive

Accepted: May 22, 2014

\section{Introduction}

Chronic obstructive pulmonary disease (COPD) is highly prevalent global cause of mortality and morbidity (1). Up to $5 \%$ of patients are genetically predisposed to premature COPD due to the alpha1-antitrypsin deficiency (AATD) (2). This condition develops as the consequence of homozygosity or compound heterozygosity for the deficiency alleles of SERPINA1, gene encoding for alpha-1-antit- rypsin (AAT). In contrast to the functional (M) alleles, these variants are associated with the reduced AAT concentration and functionality. Regarding prevalence, deficiency alleles are classified as common $(Z, S)$ or rare $\left(M_{\text {malton' }} S_{\text {iiyama' }}, M_{\text {heerlen' }} M_{\text {procida' }}\right.$ null alleles etc) (3). Timely detection of AATD allows significant health benefits like AAT augmentation and genetic counselling. Nevertheless, it is 
estimated that only a minority (4-5\%) of COPD patients with AATD are identified (2).

World Health Organization (WHO) and different pulmonologists' associations advise measurement of AAT concentration as screening in COPD generally, with the special emphasis on targeted groups (i.e. those diagnosed with COPD before the age of 45 , patients with family history of COPD). In case of decreased concentration, it is recommended to analyse patient's pheno- or genotype (3).

Laboratory methods for testing AATD are associated with different limitations. Immunonephelometric assays provide data about AAT serum concentration, whereby different acquired factors (inflammation, hepatic or renal failure) significantly limit their reliability. In phenotyping, difficulties in interpretation and technical performance of thinlayer isoelectric focusing (IEF) can be encountered. For molecular techniques caution is necessary since rare alleles might not be detected unless standard genotyping is followed by sequencing. To overcome these issues and improve effectiveness of AATD testing, several centers have integrated methods and created laboratory algorithms for AATD detection (4-6). Following their experience, integrative algorithm was proposed with intention to increase AATD detection among Serbian patients. Algorithm starts with concomitant determination of AAT concentration and genotyping for $\mathrm{Z}$ and $\mathrm{S}$ alleles. IEF is performed as the reflex test to resolve discrepancies between the AAT concentration and associated genotype-concentration bellow $0.90 \mathrm{~g} / \mathrm{L}$ and $\mathrm{MM}, 0.61 \mathrm{~g} / \mathrm{L}$ and $\mathrm{MZ}$ or 0.84 $\mathrm{g} / \mathrm{L}$ and MS genotype. Additional or bands with altered position in gel pattern indicate the presence of rare alleles, which has to be confirmed with DNA sequencing. If no specific bands are observed, the most probable cause of discrepancy could be attributed to acquired conditions affecting AAT concentration. Nevertheless, caution is needed in such situations to avoid neglecting of null allele presence and referring sample to DNA sequencing (7).

Considering the limited reliability of AAT concentration due to the effects of acquired factors, we hypothesized that an integrative algorithm could lead to the improved effectiveness by assuring
AATD detection in heterozygous carriers with AAT concentration in reference range. The aim of our study was to analyse the effectiveness of an integrative laboratory algorithm for AATD detection in patients diagnosed with COPD by the age of 45 years, in comparison with the screening approach based on AAT concentration measurement alone.

\section{Materials and methods}

\section{Study design}

This cross-sectional study included patients from the Clinic for Lung Diseases in Clinical Center of Serbia, Belgrade. They were recruited from January 2011 till December 2013. Laboratory testing was performed in the Center for Medical Biochemistry in Clinical Center of Serbia and Institute of Molecular Genetics and Genetic Engineering, University of Belgrade. The study was approved by the Ethics Committee of Clinical Center of Serbia and all patients signed written consent.

\section{Patients}

The study enrolled fifty unrelated COPD patients (28 males and 22 females, 52 (24-75) years). Included were patients with the age $\leq 45$ years at the time of COPD diagnosis in whom AATD presence was not investigated prior to inclusion in the study. COPD was diagnosed on the basis of spirometry measurements: ratio of postbronchodilator forced expiratory volume in $1 \mathrm{~s}$ (FEV1) to forced vital capacity (FVC) less than 0.7 and FEV1 less than $80 \%$ (8).

\section{Blood sampling}

Venepunction was performed in the sitting position at 8 a.m., after the overnight fasting (9). Blood was collected in two Vacutainer (BD Vacutainer, Franklin Lakes, NJ, USA) tubes - one SST ${ }^{\circledR}$ II Advance and one with $0.105 \mathrm{~mol} / \mathrm{L}$ sodium citrate. Serum was separated after centrifugation for 15 minutes at $1500 \times \mathrm{g}$ and stored at $-20^{\circ} \mathrm{C}$ for up to 4 weeks. DNA was isolated with Illustra blood genomic Prep Mini Spin $\mathrm{Kit}^{\circ}$ (GE Healthcare, Little Chalfont, UK), following manufacturer's instructions, and kept at $+4{ }^{\circ} \mathrm{C}$. 


\section{Laboratory methods}

AAT concentration was measured with immunonephelometry assay using $\mathrm{N}$ Antiserum to human a1-Antitrypsin (Siemens Healthcare Diagnostics Products $\mathrm{GmbH}$, Marburg, Germany) on BN II Nephelometer (same manufacturer). According to the manufacturer's data, method reference range is $0.90-2.00 \mathrm{~g} / \mathrm{L}$, and within-run and between-run coefficients of variation were $1.8 \%$ and $1.2 \%$ at 1.46 $\mathrm{g} / \mathrm{L}$ and $2.3 \%$ and $1.1 \%$ at $2.03 \mathrm{~g} / \mathrm{L}$ respectively.

IEF was performed on precasted polyacrylamide gels plates $(245 \times 110 \times 1 \mathrm{~mm}, \mathrm{~T}=5 \%$ \& $\mathrm{C}=3 \%$, pH gradient $=4.0-5.0)$, under the conditions recommended by the manufacturer (GE Healthcare, Little Chalfont, UK). Phenotype was analyzed through gel patterns comparison with commercial samples of known phenotype (Sebia, Evry Cedex, France).

Genotyping for $Z$ and $S$ allele was performed using the PCR-reverse ASO hybridization GenoType AAT kit (Hain Lifescience $\mathrm{GmbH}$, Nehren, Germany), according to the manufacturer's instructions. PCR-direct sequencing was performed with the following primers: $5^{\prime}$-TGTCAATCCCTGATCACT-3'/5'TTGCTTGTTTCTATGGGAAC-3'; 5'-GGTTTCTTTATTCTGCTACA-3'/5'-TCAGTCCCAACATGGCTAA-3'; 5'GGCAGAAATAATCAGAAAAG -3'/5'- TGGTATCTGTAGGGAAGA -3'; and 5'- GTGACAGGGAGGGAGAGGA -3'/5'-GGAGGGATTTACAGTCACAT-3' (Eurofins MWG Operon, Huntsville, AL, USA) for SERPINA1 exons 2, 3, 4 and 5. ABI Prism BigDye Terminator Kit v3.1 Cycle Sequencing Kit and Sequencing Analysis Software v5.2 Patch were employed according to manufacturer's instructions (Applied Biosystems, Life Technologies, Thermo Fisher Scientific brand of Waltham, Massachusetts, USA).

\section{Statistical analysis}

Absolute frequencies were presented for genotypes and ratios for allelic frequencies. AAT concentration was presented as median with range (min-max). Kruskal-Wallis and Mann-Whitney $U$ test with Boniferroni correction were used to compare AAT concentrations between groups of patients with different AATD status. Differences in number of detected AATD cases and carriers were tested using McNemar Chi-square test for dependent samples with exact $P$ module. Data analysis was performed with statistical software $\mathrm{SPSS}^{\circ}$ version 22.0 (IBM ${ }^{\oplus}$, New York, USA, trial version). P value bellow 0.05 was considered statistically significant.

\section{Results}

Data about detected genotypes and corresponding AAT concentrations are presented in Table 1. Calculated allelic frequencies were 0.2, 0.02 and 0.76 for $Z, S$ and $M$ allele respectively, while joint frequency of rare alleles was 0.02. AATD was detected in 7 and carrier state in 10 cases. AAT concentration in patients with AATD $(0.20(0.11-0.26)$ $\mathrm{g} / \mathrm{L})$ was significantly lower in comparison both with AATD carriers $(0.96(0.65-2.30) \mathrm{g} / \mathrm{L} ; \mathrm{P}<0.001)$ and patients without AATD (1.31 (0.92-2.35) g/L; P $<0.001)$. In addition, no significant difference was observed between last two groups $(P=0.125)$, whereby the half of carriers ( 3 with $M Z$ and 2 with MS genotype) had AAT concentration in the reference range.

TABLE 1. Detected genotypes and corresponding AAT concentrations.

\begin{tabular}{ccc}
\hline Genotype & $\begin{array}{c}\text { Number of } \\
\text { patients }\end{array}$ & $\begin{array}{c}\text { AAT concentration } \\
(\mathbf{g} / \mathbf{L})\end{array}$ \\
\hline $\mathrm{ZZ}$ & 5 & $0.21(0.20-0.26)$ \\
Rare & 2 & $0.16(0.11-0.20)$ \\
$\left(\mathrm{ZM}\right.$ malton' $_{\mathrm{ZQ}}$ amersfoort $)$ & 2 & $0.77(0.65-2.27)$ \\
$\mathrm{MZ}$ & 8 & $1.72(1.14-2.30)$ \\
$\mathrm{MS}$ & 2 & $1.36(0.92-2.35)$ \\
$\mathrm{MM}$ & 33 &
\end{tabular}

The results obtained in the first diagnostic line were sufficient to evaluate the presence of AATD in 48 of enrolled patients. Discrepancy between immunonephelometry and results of genotyping for $Z$ allele, was encountered in two cases.

Case 1: AAT concentration of $0.20 \mathrm{~g} / \mathrm{L}$ was matched with $M Z$ genotype. IEF pattern (Figure $1 A$ ) showed additional band positioned slightly cathodal compared with bands corresponding to M2 allele, thus indicative for $M_{\text {malton. }}$. The presumption was confirmed by sequencing (Figure 1B), leading to the 

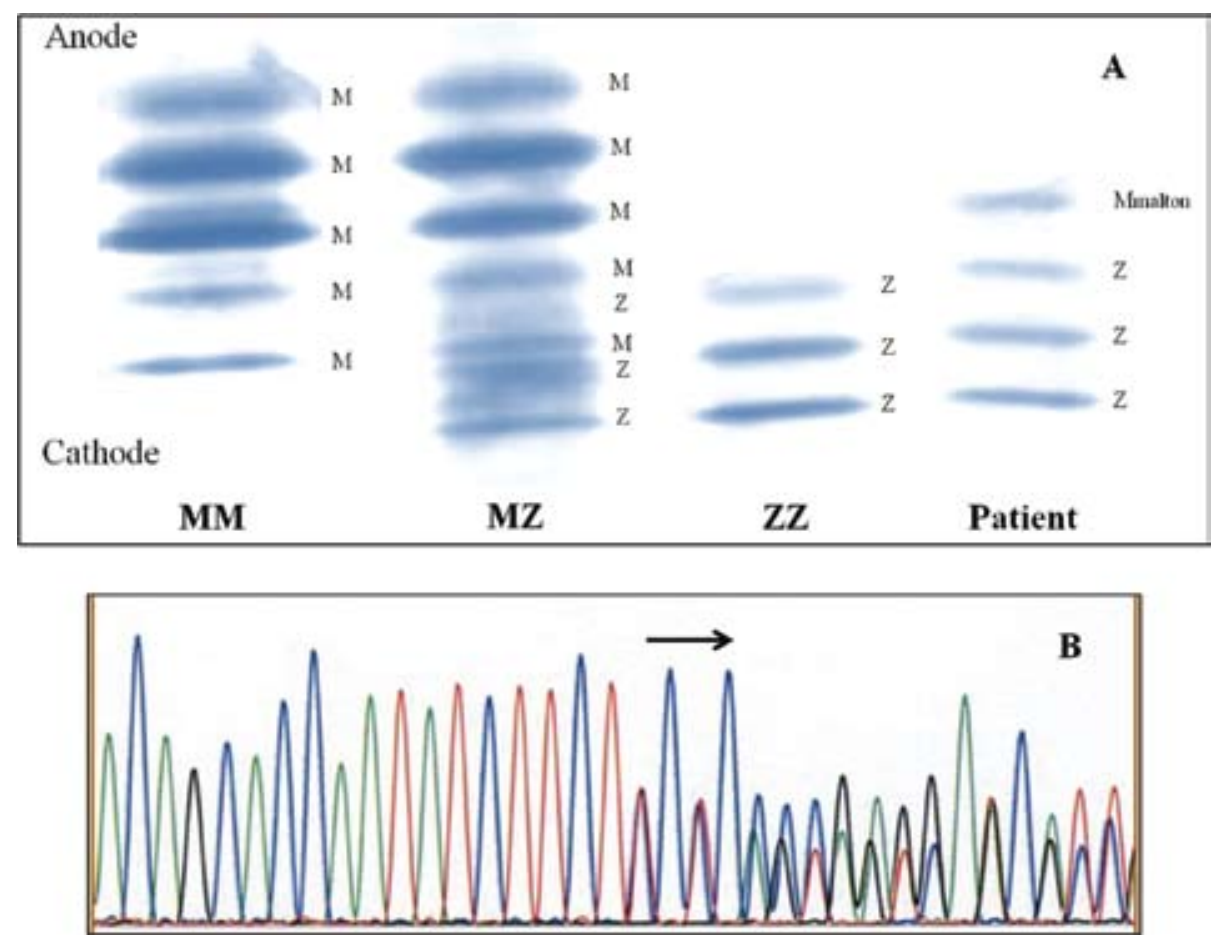

Figure 1. Identification of $M_{\text {malton }}$ allele.

$1 A$ - Determination of AAT phenotype in the patient and comparison to controls of the $M M, M Z$, and $Z Z$ phenotype.

1B - DNA sequence of part of exon 2 in the SERPINA1, showing the heterozygous form of in- frame deletion of TTC codon for Phe ${ }^{75}$ or $\mathrm{Phe}^{76}$.

conclusion that the patient was heterozygote for $\mathrm{Z}$ and $M_{\text {malton }}$ alleles.

Case 2: AAT concentration of $0.11 \mathrm{~g} / \mathrm{L}$ was measured and $M Z$ genotype detected. IEF gel pattern contained no additional bands. The presence of null allele was suspected and confirmed by sequencing, which identified $\mathrm{Q} \mathrm{O}_{\text {amersfoort }}$ (Figure 2).

The difference in effectiveness of the screening approach in this group was estimated from the obtained results. Five carriers remained undetected and two AATD (compound heterozygotes for
$Z$ and rare alleles) could not be unequivocally determined. Nevertheless, the estimated effectiveness was not significantly different from integrative algorithm both for AATD $(P=0.500)$ and carriers $(P=0.063)$.

\section{Discussion}

Our study showed that integrative algorithm revealed rather high prevalence of AATD in a group of patients with premature COPD. Majority of affected subjects inherited $Z$ allele homozygously,

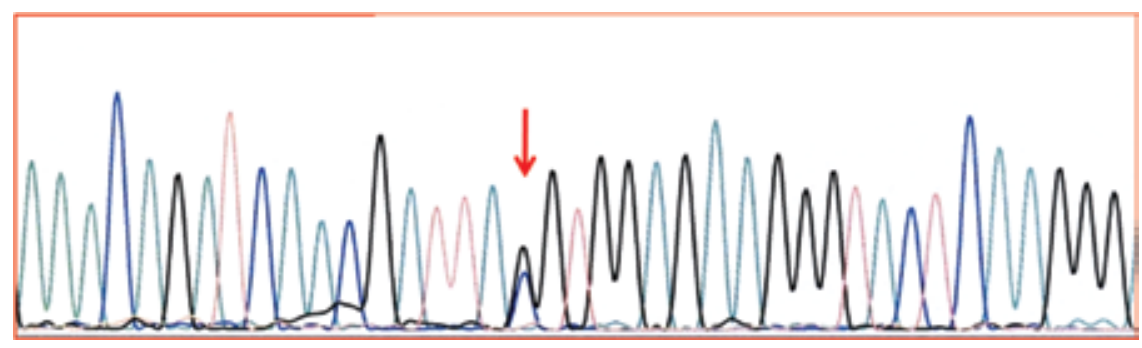

FigURE 2. Identification of $\mathrm{QO}_{\text {amersfoort }}$ allele.

DNA sequence of part of exon 2 in the SERPINA1, showing the heterozygous form of Tyr184TAC - stop184TAG. 
while compound heterozygosity with rare alleles was detected in two patients. In the half of the heterozygous carriers AAT concentration was in the reference range, indicating that they would be undetected by screening relying only on AAT concentration. Nevertheless the integrative algorithm was not shown to be more effective than screening based on AAT concentration alone, in the investigated group.

Previously reported AATD percentage in unselected COPD patients, ranging from 0.5 to 10.4 (10), was much lower than the prevalence obtained in this study. Additionally, in a group of randomly collected Serbian COPD patients AATD prevalence was found to be $2.9 \%$ (11) There is only one report on AATD prevalence among targeted COPD patients (premature emphysema, emphysema with no recognized risk factor, pneumothorax) which exceeded $40 \%$ (12). Taken together with the last example, our results might suggest that targeting on patients with premature COPD might be a useful mode to increase AATD detection rate. Nevertheless, this presumption has to be confirmed in a larger cohort of patients.

Previously reported overlapping between AAT concentrations corresponding to AATD carriers and patients with no AATD $(3,6,13)$ was evidenced in the investigated patients. Through concomitant measurement of serum AAT concentration and genotyping for $Z$ and $S$ allele, integrative algorithm diminished the possibility that carriers remain undetected. It is noteworthy that this combination also improves diagnostic accuracy because genotyping for $\mathrm{Z}$ and $\mathrm{S}$ allele, if performed alone, might miss rare alleles $(5,6,14)$. Regarding the investigated group, two patients might be erroneously classified as carriers of $Z$ allele.

The reliable detection of rare alleles is very important criterion for algorithm application (15). Their presence was confirmed in two patients, one carrying $\mathrm{ZM}_{\text {malton }}$ genotype and the other $\mathrm{ZQO}_{\text {amers- }}$ foort $M_{\text {malton }}$ is derived from the functional allele by the deletion of a TTC codon. Consequential lack of a phenylalanine residue potentiates protein aggregation in hepatocytes, while serum concentrations reach less than $15 \%$ of normal. The presence of this allele is associated with lung and liver disease (16).
According to the currently available data, the index case with $M_{\text {malton }} Z$ genotype from this study is the second reported in Serbian population (17). $\mathrm{Q0}_{\text {amersfoort }}$ is characterized by the nonsense mutation leading to the formation of TAG stop codon. Reliable data about clinical significance of this allele are not yet available (18). The index case with $\mathrm{QO}_{\text {amersfoort }} \mathrm{Z}$ genotype identified in this study is the first report about this allele in patients of Serbian origin.

Rather small and preselected group of patients, recruited in a single institution is the main limitation of the study. In addition, the presence of additional COPD risk factors (smoking, air pollution exposure) was not considered during the recruitment. For these reasons the observed positive outcomes should be regarded as "pilot". Moreover, they might explain why the integrative algorithm was not shown to be more effective than previously recommended screening approach based on AAT measurement. To resolve this issues further studies in larger cohorts of COPD patients, both unselected and targeted are recommended.

\section{Conclusion}

There is a high prevalence of AATD affected subjects and carriers in patients with premature COPD. The use of integrative laboratory algorithm does not improve the effectiveness of AATD detection in comparison with the screening based on AAT concentration alone.

\section{Acknowledgements}

The Ministry of Education, Science and Technological Development of the Republic of Serbia supported this study on the basis of contracts No. 175036, 173008 and 175034. The authors are grateful to Prof. Svetlana Ignjatovic (Center for Medical Biochemistry, Clinical Center of Serbia \& Faculty of Pharmacy, University of Belgrade, Belgrade, Serbia), Marijana Dajak, Ph.D (Center for Medical Biochemistry, Clinical Center of Serbia, Belgrade, Serbia) and Dr. Nebojsa Dovezenski (LKB Vertriebs $\mathrm{GmbH}$ ) for their valuable help, advices and suggestions.

\section{Potential conflict of interest}

None declared. 


\section{References}

1. Global initiative for Obstructive Lung Disease (GOLD). Global strategy for diagnosis, management and prevention of COPD (Updated 2014). Available at: http://www.goldcopd. org/uploads/users/files/GOLD_Report_2014_Jan23.pdf. Accessed: May 7th 2014.

2. Brode SK, Ling SC, Chapman KR. Alpha-1 antitrypsin deficiency: a commonly overlooked cause of lung disease. Can Med Assoc J 2012;184:1365-71. http://dx.doi.org/10.1503/ cmaj. 111749.

3. de Serres F, Blanco I. Role of alpha-1 antitrypsin in human health and disease. J Intern Med 2014; [Epub ahead of print]. http://dx.doi.org/10.1111/joim.12239.

4. Snyder MR, Katzmann JA, Butz ML, Yang P, Dawson DB, Kevin $\mathrm{CH}$, et al. Diagnosis of a-1-antitrypsin: an algorithm of quantification, genotyping and phenotyping. Clin Chem 2006;52:2236-42. http://dx.doi.org/10.1373/clinchem. 2006.072991.

5. Bornhorst JA, Procter M, Meadows C, Ashwood ER, Mao $R$. Evaluation of an integrative diagnostic algorithm for the identification of people at risk for a1-antitrypsin deficiency. Am J Clin Pathol 2007;128:482-90. http://dx.doi. org/10.1309/44J4KBCFQ8E9D1B8.

6. Miravitlles $M$, Herr C, Ferrarotti I, Jardi R, RodriguezFrias, Luisetti $M$, et al. Laboratory testing of individuals with severe a1-antitrypsin deficiency in three European centres. Eur Respir J 2010;35:960-8. http://dx.doi. org/10.1183/09031936.00069709.

7. Beletic A, Dudvarski-llic A, Milenkovic B, Nagorni-Obradovic $L j$, Ljujic $M$, Djordjevic V, et al. Alpha-1-antitrypsin deficiency- molecular basis, clinical presentation, therapeutic options and an integrative approach in diagnostics. J Med Biochem 2014;33:88-96. http://dx.doi.org/10.2478/jomb2013-0038.

8. Global initiative for Obstructive Lung Disease (GOLD). Global strategy for diagnosis, management and prevention of COPD (Updated 2010). Available at: http://www.goldcopd.org/uploads/users/files/GOLDReport_April112011.pdf. Accessed: May 7th 2014.

9. Nikolac N, Supak-Smolcić V, Simundić AM, Celap I. Croatian Society of Medical Biochemistry and Laboratory Medicine: national recommendations for venous blood sampling. Biochem Med 2013;23:242-54. http://dx.doi.org/10.11613/ BM.2013.031.
10. Stoller JK, Brantly M. The challenge of detecting alpha-1 antitrypsin deficiency. COPD 2013;10(S1):26-34. http://dx.doi. org/10.3109/15412555.2013.763782.

11. Topic A, Stankovic M, Divac-Rankov A, Petrovic-Stanojevic $N$, Mitic-Milikic M, Nagorni-Obradovic L, Radojkovic D. Alpha-1-antitrypsin deficiency in Serbian adults with lung diseases. Genet Test Mol Biomarkers 2012;16:1282-6. http:// dx.doi.org/10.1089/gtmb.2012.0152.

12. Corda L, Bertella E, Pini L, Pezzini A, Medicina D, Boni E, et al. Diagnostic flow chart for targeted detection of alpha1antitrypsin deficiency. Resp Med 2006;100:463-70. http:// dx.doi.org/10.1016/j.rmed.2005.06.009.

13. Donato $L$, Jenkins $S M$, Smith C, Katzmann JA, Snyder MR. Reference and interpretive ranges for a(1)-antitrypsin quantitation by phenotype in adult and pediatric populations. Am J Clin Pathol 2012;138:398-405. http://dx.doi. org/10.1309/AJCPMEEJK32ACYFP.

14. Bals $R$, Koczulla $R$, Kotke V, Andress J, Blackert K, Vogelmeier $C$. Identification of individuals with alpha-1-antitrypsin deficiency by a targeted screening program. Resp Med 2007;101:1708-14. http://dx.doi.org/10.1016/j. rmed.2007.02.024.

15. Rodriguez-Frias F, Miravitlles M, Vidal R, Camos S, Jardi $R$. Rare alpha-1-antitrypsin variants: are they really so rare? Ther Adv Respir Dis 2012;6:79-85. http://dx.doi. org/10.1177/1753465811434320.

16. Salahuddin P. Genetic Variants of a1-Antitrypsin. Curr Prot Pept Sc 2010;11:101-7. http://dx.doi.org/10.2174/ 138920310790848368.

17. Topic A, Jelic Z, Ilic A. A case of severe alpha-1 antitrypsin deficiency associated with the rare Pi MmaltonZ genotype. Balkan J Clin Lab 1995;2:95.

18. Prins $J$, van der Meijden BB, Kraaijenhagen $R J$, Wielders JPM. Inherited Chronic Obstructive Pulmonary Disease: New Selective-Sequencing Workup for a1-Antitrypsin Deficiency Identifies 2 Previously Unidentified Null Alleles. Clin Chem 2008;54:101-7. http://dx.doi.org/10.1373/ clinchem.2007.095125. 